\title{
MOv19 CAR-CD3zeta-4-1BB-expressing Allogeneic T-lymphocytes
}

National Cancer Institute

\section{Source}

National Cancer Institute. MOv19 CAR-CD3zeta-4-1BB-expressing Allogeneic Tlymphocytes. NCI Thesaurus. Code C162166.

A preparation of allogeneic CD4-positive and CD8-positive T-lymphocytes that have been transduced with a lentiviral vector expressing a chimeric antigen receptor (CAR) comprised of an anti-folate receptor alpha (FR-alpha; ovarian tumor-associated antigen MOv18) single chain variable fragment (scFv) (MOv19) and the zeta chain of the T-cell receptor (TCR)/CD3 complex (CD3-zeta), coupled to the signaling domain of 4-1BB (CD137), with potential immunomodulating and antineoplastic activities. Upon administration, MOv19-CAR-CD3zeta-4-1BB-expressing allogeneic T-lymphocytes target, bind to and induce selective toxicity in folate receptor alpha-expressing tumor cells. The costimulatory signaling domain enhances both proliferation of T-cells and antitumor activity. Folate receptor alpha is a glycosylphosphatidylinositol-linked cellsurface glycoprotein that is widely expressed in certain cancers including serous and epithelial ovarian cancer, endometrial adenocarcinoma, non-small cell lung cancer and triple negative breast cancer. In contrast, folate receptor alpha expression is limited in normal tissues. 